\title{
Extended Cesáro operators between generalized Besov spaces and Bloch type spaces in the unit ball
}

\author{
Ze-Hua Zhou and Min Zhu
}

(Communicated by Hans G. Feichtinger)

2000 Mathematics Subject Classification. Primary: 47B38; Secondary: $46 \mathrm{E} 15,32 \mathrm{~A} 37$.

Keywords and phrases. Generalized Besov space, Bloch-type space, extended Cesáro operators, boundedness, compactness.

\begin{abstract}
Let $g$ be a holomorphic of the unit ball $B$ in the $n$-dimensional complex space, and denote by $T_{g}$ the extended Cesá ro operator with symbol $g$. Let $0<p<+\infty,-n-1<q<+\infty, q>-1$ and $\alpha>0$, starting with a brief introduction to well known results about Cesáro operator, we investigate the boundedness and compactness of $T_{g}$ between generalized Besov space $B(p, q)$ and $\alpha$-Bloch space $\mathcal{B}^{\alpha}$ in the unit ball, and also present some necessary and sufficient conditions.
\end{abstract}

\section{Introduction}

For any $z=\left(z_{1}, \cdots, z_{n}\right), \quad w=\left(w_{1}, \cdots, w_{n}\right) \in C^{n}$, the inner product is defined by $\langle z, w\rangle=\sum_{k=1}^{n} z_{k} \bar{w}_{k}$. Let $B$ be the unit ball of $C^{n}$, the class of all holomorphic functions on $B$ is defined by $H(B)$. For $f \in H(B)$, we write

$$
\nabla f(z)=\left(\frac{\partial f}{\partial z_{1}}(z), \cdots, \frac{\partial f}{\partial z_{n}}(z)\right)
$$


and

$$
R f(z)=\langle\nabla f(z), \bar{z}\rangle=\sum_{j=1}^{n} z_{j} \frac{\partial f(z)}{\partial z_{j}} .
$$

For $\alpha \geq 0, f$ is said to be in the Bloch space $\mathcal{B}^{\alpha}$ provided that $f \in H(B)$ and

$$
\|f\|_{\alpha}=\sup _{z \in B}\left(1-|z|^{2}\right)^{\alpha}|\nabla f(z)|<+\infty .
$$

As we all know, $\mathcal{B}^{\alpha}$ is a Banach space when $\alpha \geq 1$ under the norm $\|f\|_{\mathcal{B}^{\alpha}}=$ $|f(0)|+\|f\|_{\alpha}$. The spaces $\mathcal{B}^{1}$ and $\mathcal{B}^{\alpha}(0<\alpha<1)$ are just the Bloch space and the Lipschitz spaces $L_{1-\alpha}$ respectively. From [26] we know that a holomorphic function $f \in \mathcal{B}^{\alpha}$ if and only if $\sup _{z \in B}\left(1-|z|^{2}\right)^{\alpha}|R f(z)|<+\infty$.

Furthermore, by the Norm Equivalent Theorem we have

$$
\|f\|_{\mathcal{B}^{\alpha}} \approx|f(0)|+\sup _{z \in B}\left(1-|z|^{2}\right)^{\alpha}|R f(z)|,
$$

where $M \approx N$ means that the two quantities $M$ and $N$ are comparable, that is, there exist two positive constants $C_{1}$ and $C_{2}$ such that $C_{1} M \leq$ $N \leq C_{2} M$.

Let $d v$ be the Lebesegue measure on the unit ball $B$ of $C^{n}$ normalized so that $v(B)=1$. For $a \in B$, let $g(z, a)=\log \left|\varphi_{a}(z)\right|^{-1}$ be the Green's function on B with logarithmic singularity at $a$, where $\varphi_{a}$ is the Möbius transformation of $B$ with $\varphi_{a}(0)=a, \varphi_{a}(a)=0, \varphi_{a}=\varphi_{a}^{-1}$.

Let $0<p, s<+\infty,-n-1<q<+\infty$ and $q+s>-1$. We say $f \in F(p, q, s)$ provided that $f \in H(B)$ and

$$
\|f\|_{F(p, q, s)}=|f(0)|+\left\{\sup _{a \in B} \int_{B}|\nabla f(z)|^{p}\left(1-|z|^{2}\right)^{q} g^{s}(z, a) d v(z)\right\}^{\frac{1}{p}}<+\infty .
$$

The space $F(p, q, s)$ was first defined in [31] (see also [27]).

Let $0<p<+\infty,-n-1<q<+\infty$ and $q>-1$. We say that $f \in B(p, q)$ if $f \in H(B)$ and

$$
\|f\|_{(p, q)}=\left\{\int_{B}|\nabla f(z)|^{p}\left(1-|z|^{2}\right)^{q} d v(z)\right\}^{\frac{1}{p}}<+\infty .
$$

It is obvious that $B(p, q)$ is a classical Besov space if we take special parameters $p, q$. It is not hard to show that $B(p, q)$ is a Banach space under the norm $\|f\|_{B(p, q)}=|f(0)|+\|f\|_{(p, q)}$, we refer the reader to see Zhu's book [35]. From Exercises 2.2 in [35], we know that a holomorphic function $f \in B(p, q)$ if and only if $\int_{B}|R f(z)|^{p}\left(1-|z|^{2}\right)^{q}<+\infty$. 
Let $f(z)$ be a holomorphic function on the unit disc $D$ with Taylor expansion $f(z)=\sum_{j=0}^{\infty} a_{j} z^{j}$, the classical Cesáro operator acting on $f$ is defined by

$$
\mathcal{C}[f](z)=\sum_{j=0}^{\infty}\left(\frac{1}{j+1} \sum_{k=0}^{j} a_{k}\right) z^{j} .
$$

Despite the simplicity of the definition of $\mathcal{C}[f](z)$, several problems are encountered when characterizing the boundedness and compactness of Cesáro operator between spaces of holomorphic functions. These problems require profound and interesting analytical machinery. Moreover, the study of Cesáro operator has arguably become a major driving force in the development of modern complex analysis. The papers listed in the bibliography are excellent sources for the recent developments in the theory of Cesáro operators. It is well known that the operator $\mathcal{C}$ is bounded on the usual Hardy spaces $H^{p}(D)$ for $0<p<\infty$, Bergman spaces and Dirichlet spaces. See $[3,7,19,20,17,25]$.

But the operator $\mathcal{C}$ is not always bounded, in [22], Shi and Ren gave a necessary and sufficient condition for the operator $\mathcal{C}$ to be bounded on mixed norm spaces in the unit disc. It is natural to ask what are the conditions for higher dimensional case.

A little calculation shows $\mathcal{C}[f](z)=\frac{1}{z} \int_{0}^{z} f(t)\left(\log \frac{1}{1-t}\right)^{\prime} d t$. From this point of view, if $g \in H(B)$, it is natural to consider the extended Cesá ro operator (also called Volterra-type operator or Riemann-Stieltjes type operator) $T_{g}$ on $H(B)$ defined by

$$
T_{g}(f)(z)=\int_{0}^{1} f(t z) R g(t z) \frac{d t}{t} .
$$

It is easy to show that $T_{g}$ take $H(B)$ into itself. In general, there is no easy way to determine when an extended Cesáro operator is bounded or compact.

The boundedness and compactness of this operator on weighted Bergman, mixed norm, Bloch, and Dirichlet spaces in the unit ball have been studided by Xiao [25], Hu and Zhang [9, 10, 11, 30], Li and Stević [12, 13, 15]. More recently, Li and Stević [14] discuss the compactness of the operator between $F(p, q, s)$ and $\alpha$-Bloch spaces in the ball, where $s$ must be positive.

In this paper, we will continue this line of research and characterize those $g$ for which $T_{g}$ is bounded (or compact) between generalized Besov space $B(p, q)$ and $\alpha$-Bloch space $\mathcal{B}^{\alpha}$ in the unit ball, and also present some necessary and sufficient conditions. For the proof, we need different method and some complex calculation skills. 
For $p>0, z \in B$, denote the function

$$
G_{p}(z)= \begin{cases}1, & 0<p<1 ; \\ \log \frac{2}{1-|z|^{2}}, & p=1 ; \\ \left(\frac{1}{1-|z|^{2}}\right)^{\alpha-1}, & p>1 .\end{cases}
$$

The main results of the paper are the following:

Theorem 1. For $0<p<+\infty,-n-1<q<+\infty, q>-1, \alpha \geq 0$, $g \in H(B), T_{g}$ is bounded from $B(p, q)$ to $\mathcal{B}^{\alpha}$ if and only if

$$
\sup _{z \in B}\left(1-|z|^{2}\right)^{\alpha} G_{\frac{n+1+q}{p}}(z)|R g(z)|<\infty .
$$

Theorem 2. For $0<p<+\infty,-n-1<q<+\infty, q>-1, \alpha \geq 0$, $g \in H(B), T_{g}$ is compact from $B(p, q)$ to $\mathcal{B}^{\alpha}$ if and only if $g \in \mathcal{B}^{\alpha}$ for $0<\frac{n+1+q}{p}<1$, and $\lim _{|z| \rightarrow 1^{-}}\left(1-|z|^{2}\right)^{\alpha} G_{\frac{n+1+q}{p}}(z)|R g(z)|=0$ for $\frac{n+1+q}{p} \geq 1$.

\section{Some Lemmas}

In the following, we will use the symbol $c$ or $C$ to denote a finite positive number which does not depend on variable $z$ and may depend on some norms and parameters $p, q, n, \alpha, x, f$ etc, not necessarily the same at each occurrence. We first give some lemmas.

Lemma 1. If $0<p<+\infty,-n-1<q<+\infty, q>-1$, then $B(p, q) \subset \mathcal{B}^{\frac{n+1+q}{p}}$ and there exists $c>0$, such that for all $f \in B(p, q)$,

$$
\|f\|_{\mathcal{B}^{\frac{n+1+q}{p}}} \leq c\|f\|_{B(p, q)} .
$$

Proof. Suppose $f \in B(p, q)$. Fix $r_{0}$ with $0<r_{0}<1$. Since $(R f) \circ \varphi_{a} \in H(B),\left|(R f) \circ \varphi_{a}\right|^{p}$ is subharmonic in $B$. That is

$$
\begin{aligned}
|R f(a)|^{p} & =\left|(R f) \circ \varphi_{a}(0)\right|^{p} \leq \frac{1}{r_{0}^{2 n}} \int_{r_{0} B}\left|(R f) \circ \varphi_{a}(\omega)\right|^{p} d v(\omega) \\
& =\frac{1}{r_{0}^{2 n}} \int_{\varphi_{a}\left(r_{0} B\right)}|(R f(z))|^{p} \frac{\left(1-|a|^{2}\right)^{n+1}}{|1-<z, a>|^{(2 n+2)}} d v(z) .
\end{aligned}
$$

From (5) in [37], we have

$$
\frac{1-r_{0}}{1+r_{0}}\left(1-|a|^{2}\right) \leq\left(1-|z|^{2}\right) \leq \frac{1+r_{0}}{1-r_{0}}\left(1-|z|^{2}\right)
$$


as $z \in \varphi_{a}\left(r_{0} B\right)$. Thus

$$
\frac{\left(1-|a|^{2}\right)^{n+1}}{|1-<z, a>|^{2 n+2}\left(1-|z|^{2}\right)^{q}} \leq \frac{4^{n+1}}{\left(1-|a|^{2}\right)^{n+1+q}}\left(\frac{1+r_{0}}{1-r_{0}}\right)^{|q|} .
$$

Consequently, we have

$$
\begin{aligned}
|R f(a)|^{p} & \leq \frac{1}{r_{0}^{2 n}} \int_{\varphi_{a}\left(r_{0} B\right)}|R f(z)|^{p} \frac{\left(1-|a|^{2}\right)^{n+1}}{|1-<z, a>|^{2 n+2}} d v(z) \\
& =\frac{1}{r_{0}^{2 n}} \int_{\varphi_{a}\left(r_{0} B\right)}|R f(z)|^{p}\left(1-|z|^{2}\right)^{q} \frac{\left(1-|a|^{2}\right)^{n+1}}{|1-<z, a>|^{2 n+2}\left(1-|z|^{2}\right)^{q}} d v(z) \\
& \leq \frac{4^{n+1} r_{0}^{-2 n}}{\left(1-|a|^{2}\right)^{n+1+q}}\left(\frac{1+r_{0}}{1-r_{0}}\right)^{|q|}\|f\|_{B(p, q)}^{p} .
\end{aligned}
$$

This shows that $f \in \mathcal{B}^{\frac{n+1+q}{p}}$ and $\|f\|_{\mathcal{B}^{\frac{n+1+q}{p}}} \leq c\|f\|_{B(p, q)}$.

Lemma 2. Let $p>0$. Then there exits a constant $c>0$ such that for all $f \in \mathcal{B}^{p}$ and $z \in B$, the estimate

$$
|f(z)| \leq c G_{p}(z)\|f\|_{\mathcal{B}^{p}},
$$

holds, where $G_{p}(z)$ is the function defined in the introduction.

Proof. This Lemma can be easily obtained by some integral estimates. See Lemma 2.2 in [27] for details.

Lemma 3. ([30]) Let $0<p<1,\left\{f_{j}\right\}$ be any bounded sequence in $\mathcal{B}^{p}$ and $f_{j}(z) \rightarrow 0$ on any compact subset of $B$. Then

$$
\lim _{j \rightarrow \infty} \sup _{z \in B}\left|f_{j}(z)\right|=0 .
$$

Lemma 4. There exists a constant $c>0$ such that for all $t>-1$ and $z \in B$,

$$
\int_{B}\left|\log \frac{1}{1-<z, w>}\right|^{2} \frac{\left(1-|w|^{2}\right)^{t}}{(1-<z, w>)^{n+1+t}} d v(w) \leq C\left(\log \frac{1}{1-|z|^{2}}\right)^{2} .
$$

Proof. This Lemma can be proved by Stirling formula and some complex integral estimates. For the convenience of the readers, we will provide the proof here. 
Denote the left term as $I_{t}$ and let $2 \lambda=t+n+1$. By Taylor expansion

$$
\left|\log \frac{1}{1-<z, w>}\right|^{2}=\sum_{u, v=1}^{+\infty} \frac{<z, w>^{u}<w, z>^{v}}{u v}
$$

and

$$
\frac{1}{|1-<z, w>|^{2 \lambda}}=\sum_{k, l=0}^{+\infty} \frac{\Gamma(\lambda+k) \Gamma(\lambda+l)}{k ! l ! \Gamma(\lambda)^{2}}<z, w>^{k}<w, z>^{l} .
$$

Therefore

$$
\begin{aligned}
I_{t} & =\int_{B} \sum_{u, v=1}^{+\infty} \sum_{k, l=0}^{+\infty} \frac{\Gamma(\lambda+k) \Gamma(\lambda+l)}{u v k ! l ! \Gamma(\lambda)^{2}}<z, w>^{k+u}<w, z>^{l+v}\left(1-|w|^{2}\right)^{t} d v(w) \\
& =\sum_{u=1}^{+\infty} \sum_{k=0}^{+\infty} \sum_{l=0}^{u+k-1} \frac{\Gamma(\lambda+k) \Gamma(\lambda+l)}{u(u+k-l) k ! l ! \Gamma(\lambda)^{2}} \int_{B}|<z, w>|^{2(u+k)}\left(1-|w|^{2}\right)^{t} d v(w)
\end{aligned}
$$

Without any loss of generality, we may assume that $z=|z| e_{1}$, so that

$$
\begin{aligned}
& \int_{B}|<z, w>|^{2(u+k)}\left(1-|w|^{2}\right)^{t} d v(w) \\
& =\int_{B}\left(|z| w_{1}\right)^{2(u+k)}\left(1-|w|^{2}\right)^{t} d v(w) \\
& =2 n \int_{0}^{1} \int_{\partial B} \rho^{2 n-1}|z|^{2(u+k)}\left|\rho \xi_{1}\right|^{2(u+k)}\left(1-\rho^{2}\right)^{t} d \rho d \delta_{n}(\xi) \\
& =2 n|z|^{2(u+k)} \int_{0}^{1} \rho^{2(u+k+n-1)+1}\left(1-\rho^{2}\right)^{t} d \rho \int_{\partial B}\left|\xi_{1}\right|^{2(u+k)} d \delta(\xi) \\
& =n|z|^{2(u+k)} \frac{\Gamma(u+k+n) \Gamma(t+1)}{\Gamma(u+k+n+t+1)} \frac{(n-1) !(u+k) !}{(u+k+n-1) !} \\
& =\frac{\Gamma(t+1) \Gamma(u+k+1) n !}{\Gamma(2 \lambda+u+k)}|z|^{2(u+k)},
\end{aligned}
$$

which gives

$$
\begin{aligned}
I_{t} & =\sum_{u=1}^{+\infty} \sum_{k=0}^{+\infty} \sum_{l=0}^{u+k-1} \frac{\Gamma(\lambda+k) \Gamma(\lambda+l)}{u(u+k-l) k ! l ! \Gamma(\lambda)^{2}} \frac{\Gamma(t+1) \Gamma(u+k+1) n !}{\Gamma(2 \lambda+u+k)}|z|^{2(u+k)} \\
& =\sum_{u=1}^{+\infty} \sum_{k=0}^{+\infty} \frac{n ! \Gamma(t+1) \Gamma(\lambda+k) \Gamma(u+k+1)}{u k ! \Gamma(\lambda)^{2} \Gamma(2 \lambda+u+k)} \sum_{l=0}^{u+k-1} \frac{\Gamma(\lambda+l)}{(u+k-l) l !}|z|^{2(u+k)}
\end{aligned}
$$




$$
\begin{aligned}
= & \sum_{u=1}^{+\infty} \sum_{k=1}^{+\infty} \frac{n ! \Gamma(t+1) \Gamma(\lambda+k) \Gamma(u+k+1)}{u k ! \Gamma(\lambda)^{2} \Gamma(2 \lambda+u+k)} \sum_{l=0}^{u+k-1} \frac{\Gamma(\lambda+l)}{(u+k-l) l !}|z|^{2(u+k)} \\
& +\sum_{u=1}^{+\infty} \frac{n ! \Gamma(t+1) \Gamma(u+1)}{u \Gamma(\lambda) \Gamma(2 \lambda+u)} \sum_{l=0}^{u-1} \frac{\Gamma(\lambda+l)}{(u-l) l !}|z|^{2 u} \\
= & I_{1}+I_{2} .
\end{aligned}
$$

By Stirling formula, there exists an absolute constant $C_{1}$ such that for all $l, u, k \geq 1$

$$
\begin{gathered}
\frac{\Gamma(\lambda+l)}{l !} \leq C_{1} l^{\lambda-1}, \quad \frac{\Gamma(u+k+1)}{\Gamma(2 \lambda+u+k)} \leq C_{1}(u+k)^{1-2 \lambda} \\
\frac{\Gamma(u+k+1)}{\Gamma(2 \lambda+u)} \leq C_{1} u^{1-2 \lambda}, \quad \frac{\Gamma(\lambda+k)}{k !} \leq C_{1} k^{\lambda-1}
\end{gathered}
$$

so that

$$
I_{1} \leq C_{1}^{3} \sum_{u=1}^{+\infty} \sum_{k=1}^{+\infty} \frac{n ! \Gamma(t+1) k^{\lambda-1}(u+k)^{1-2 \lambda}}{u \Gamma(\lambda)^{2}} \sum_{l=1}^{u+k-1} \frac{l^{\lambda-1}}{(u+k-l)}|z|^{2(u+k)}
$$

and

$$
I_{2} \leq C_{1}^{2} \sum_{u=1}^{+\infty} \frac{n ! \Gamma(t+1) u^{1-2 \lambda}}{u \Gamma(\lambda)} \sum_{l=1}^{u-1} \frac{l^{\lambda-1}}{(u-l)}|z|^{2 u}
$$

Note that

$$
\sum_{l=1}^{M-1} \frac{l^{(\lambda-1)}}{M-l} \approx M^{\lambda-2} \log M
$$

for any $M \geq 2$. Consequently, there exists a constant $C$, such that

$$
\begin{aligned}
I_{1} & \leq C \sum_{u=1}^{+\infty} \sum_{k=1}^{+\infty} \frac{n ! \Gamma(t+1) k^{\lambda-1}(u+k)^{1-2 \lambda}}{\Gamma(\lambda)^{2} u}(u+k)^{\lambda-2} \log (u+k)|z|^{2(u+k)} \\
& =C \sum_{u=1}^{+\infty} \sum_{k=1}^{+\infty} \frac{n ! \Gamma(t+1)}{\Gamma(\lambda)^{2}} \frac{k^{\lambda}}{(u+k)^{\lambda}} \frac{\log (u+k)}{u+k} \frac{1}{u k}|z|^{2(u+k)} \\
& \leq C \sum_{u=1}^{+\infty} \sum_{k=1}^{+\infty} \frac{1}{u k}|z|^{2(u+k)}=C\left(\log \frac{1}{1-|z|^{2}}\right)^{2}
\end{aligned}
$$

and 


$$
\begin{aligned}
I_{2} & \leq C \sum_{u=1}^{+\infty} \frac{n ! \Gamma(t+1) u^{1-2 \lambda}}{\Gamma(\lambda) u} u^{\lambda-2} \log u|z|^{2 u} \\
& =C \sum_{u=1}^{+\infty} \frac{n ! \Gamma(t+1)}{\Gamma(\lambda)} \frac{1}{u^{\lambda+1}} \frac{\log u}{u}|z|^{2 u}
\end{aligned}
$$

Clearly, $I_{2}$ can be controlled by $\left(\log \frac{1}{1-|z|^{2}}\right)^{2}$ and the assertion is proved.

Lemma 5. Let $g$ be a holomorphic self-map of $B, K$ is an arbitrary compact subset of $B$. Then $T_{g}: B(p, q) \rightarrow \mathcal{B}^{\alpha}$ is compact if and only if for any uniformly bounded sequence $\left\{f_{j}\right\}(j \in N)$ in $B(p, q)$ which converges to zero uniformly for $z$ on $K$ when $j \rightarrow \infty,\left\|T_{g} f_{j}\right\|_{\mathcal{B}^{\alpha}} \rightarrow 0$ holds.

Proof. Assume that $T_{g}$ is compact and suppose $\left\{f_{j}\right\}$ is a sequence in $B(p, q)$ with $\sup _{j \in N}\left\|f_{j}\right\|_{B(p, q)}<\infty$ and $f_{j} \rightarrow 0$ uniformly on compact subsets of $B$. By the compactness of $T_{g}$ we have that $\left\{T_{g} f_{j}\right\}$ has a subsequence $\left\{T_{g} f_{j_{m}}\right\}$ which converges in $\mathcal{B}^{\alpha}$, say, to $h$. By Lemma 2, it follows that for any compact set $K \subset B$, there is a positive constant $C_{K}$ independent of $f$ such that $\left|T_{g} f_{j}(z)-h(z)\right| \leq C_{K}\left\|T_{g} f_{j}-h\right\|_{\beta^{\alpha}}$ for all $z \in K$. This implies that $T_{g} f_{j}(z)-h(z) \rightarrow 0$ uniformly on compact sets of $B$. Since $K$ is a compact subset of $B$, by the hypothesis and the definition of $T_{g}, T_{g} f_{j}(z)$ converges to zero uniformly on $K$. Since $K$ is arbitrary, it follows that the limit function $h$ is equal to 0 . Since it is true for arbitrary subsequence of $\left\{f_{j}\right\}$, we see that $T_{g} f_{j} \rightarrow 0$ in $\mathcal{B}^{\alpha}$.

Conversely, let $\left\{f_{j}\right\} \in K_{r}=B_{B(p, q)}(0, r)$, where $B_{B(p, q)}(0, r)$ is a ball in $B(p, q)$. Then by Lemma $2,\left\{f_{j}\right\}$ is uniformly bounded in arbitrary compact subset $M$ of $B$. By Montel's Lemma, $\left\{f_{j}\right\}$ is a normal family, therefore there is a subsequence $\left\{f_{j_{m}}\right\}$ which converges uniformly to $f \in H(B)$ on compact subsets of $B$. It follows that $\nabla f_{j_{m}} \rightarrow \nabla f$ uniformly on compact subsets of $B$.

Denote $B_{k}=B\left(0,1-\frac{1}{k}\right) \subset C^{n}$, then

$$
\begin{aligned}
\int_{B}|\nabla f|^{p}\left(1-|z|^{2}\right)^{q} d v(z) \\
\quad=\lim _{k \rightarrow+\infty} \int_{B_{k}} \lim _{m \rightarrow+\infty}\left|\nabla f_{j_{m}}\right|^{p}\left(1-|z|^{2}\right)^{q} d v(z) \\
\quad \leq \lim _{k \rightarrow+\infty} \lim _{m \rightarrow+\infty} \int_{B_{k}}\left|\nabla f_{j_{m}}\right|^{p}\left(1-|z|^{2}\right)^{q} d v(z) .
\end{aligned}
$$


But $\left\{f_{j_{m}}\right\} \subset B_{B(p, q)}(0, r)$. Therefore

$$
\int_{B_{k}}\left|\nabla f_{j_{m}}\right|^{p}\left(1-|z|^{2}\right)^{q} d v(z)<r^{p}
$$

so that

$$
\int_{B}|\nabla f|^{p}\left(1-|z|^{2}\right)^{q} d v(z) \leq r^{p} .
$$

Consequently, $\|f\|_{B(p, q)} \leq r$, and $f \in B(p, q)$. Hence $\left\|f_{j_{m}}-f\right\| \leq 2 r<\infty$ and the sequence $\left\{f_{j_{m}}-f\right\}$ converges to 0 on compact subsets of $B$, by the hypothesis of this lemma, it follows that $T_{g} f_{j_{m}} \rightarrow T_{g} f$ in $\mathcal{B}^{\alpha}$. Thus the set $T_{g}\left(K_{r}\right)$ is relatively compact and the proof is complete.

Lemma 6. Let $g \in H(B)$, then for any $f \in H(B)$ and $z \in B$

$$
R\left[T_{g} f\right](z)=f(z) R g(z) .
$$

Proof. Suppose the holomorphic function $f R g$ has the Taylor expansion

$$
(f R g)(z)=\sum_{|\alpha| \geq 1} a_{\alpha} z^{\alpha}
$$

Then we have

$$
\begin{aligned}
R\left(T_{g} f\right)(z) & =R \int_{0}^{1} f(t z) R(t z) \frac{d t}{t}=R \int_{0}^{1} \sum_{|\alpha| \geq 1} a_{\alpha}(t z)^{\alpha} \frac{d t}{t} \\
& =R\left[\sum_{|\alpha| \geq 1} \frac{a_{\alpha} z^{\alpha}}{|\alpha|}\right]=\sum_{|\alpha| \geq 1} a_{\alpha} z^{\alpha}=(f R g)(z) .
\end{aligned}
$$

\section{Proof of Theorem 1}

Suppose $\sup _{z \in B}\left(1-|z|^{2}\right)^{\alpha} G_{\frac{n+1+q}{n}}(z)|R g(z)|<\infty$. By Lemmas 1, 2 and 6 , it follows that for all $f \in H(B)^{p}$

$$
\begin{aligned}
\left(1-|z|^{2}\right)^{\alpha}\left|R\left[T_{g} f\right](z)\right| & =\left(1-|z|^{2}\right)^{\alpha}|f(z) \| R g(z)| \\
& \leq c\left(1-|z|^{2}\right)^{\alpha} G_{\frac{n+1+q}{p}}(z)|R g(z)| \\
& \leq c\|f\|_{B(p, q)}\left(1-|z|^{2}\right)^{\alpha} G_{\frac{n+1+q}{p}}(z)|R g(z)| \\
& \leq c\|f\|_{B(p, q)}
\end{aligned}
$$


which implies that $T_{g}$ is bounded. On the other hand, suppose $T_{g}$ is bounded, with $\left\|T_{g} f\right\|_{\mathcal{B}^{\alpha}} \leq c\|f\|_{B(p, q)}$.

Case 1. If $0<\frac{n+1+q}{p}<1$, it is clear that $T_{g} f \in \mathcal{B}^{\alpha}$ for $f=1$, i.e.,

$$
\sup _{z \in B}\left(1-|z|^{2}\right)^{\alpha}\left|R T_{g} f(z)\right|=\sup _{z \in B}\left(1-|z|^{2}\right)^{\alpha}|R g(z)|<\infty .
$$

Case 2. If $\frac{n+1+q}{p}>1$, we need to prove that

$$
\sup _{z \in B}\left(1-|z|^{2}\right)^{\alpha}\left(\frac{1}{1-|z|^{2}}\right)^{\frac{n+1+q}{p}-1}|R g(z)|<\infty .
$$

For $w \in B$, consider the test function

$$
f_{w}(z)=\frac{1-|w|^{2}}{(1-<z, w>)^{\frac{n+1+q}{p}}} .
$$

It is easy to see that

$$
\int_{B}\left(1-|z|^{2}\right)^{q}\left|\nabla f_{w}(z)\right|^{p} d v(z) \leq c\left(1-|w|^{2}\right)^{p} \int_{B} \frac{\left(1-|z|^{2}\right)^{q}}{|1-<z, w>|^{n+1+q+p}} d v(z) \leq c .
$$

The last inequality follws from [35], so that $f_{w} \in B(p, q)$ for any $w \in B$. With the boundedness of $T_{g}$, we get

$$
\begin{aligned}
\left(1-|z|^{2}\right)^{\alpha}\left(\frac{1}{1-|z|^{2}}\right)^{\frac{n+1+q}{p}-1}|R g(z)| \\
=\left(1-|z|^{2}\right)^{\alpha}\left|f_{z}(z)\right||R g(z)| \\
=\left(1-|z|^{2}\right)^{\alpha}\left|R\left(T_{g} f_{z}\right)(z)\right| \\
\leq\left\|T_{g} f_{z}\right\|_{\mathcal{B}^{\alpha}} \leq c\left\|T_{g}\right\|<\infty .
\end{aligned}
$$

Case 3. If $\frac{n+1+q}{p}=1$, namely $p=n+1+q$, we need to prove

$$
\sup _{z \in B}\left(1-|z|^{2}\right)^{\alpha} \log \frac{2}{1-|z|^{2}}|R g(z)|<\infty .
$$

For $w \in B$, consider the test function

$$
f_{w}(z)=\left(\log \frac{1}{1-|w|^{2}}\right)^{-\frac{2}{p}}\left(\log \frac{1}{1-<z, w>}\right)^{1+\frac{2}{p}} .
$$

It is easy to show that $f_{w} \in B(p, q)$ from Lemma 4 . The result follows by the same discussion as that of Case 2, the detail is omitted. So, the proof of Theorem 1 is completed. 


\section{Proof of Theorem 2}

Case 1. $0<\frac{n+1+q}{p}<1$. If $T_{g}$ is compact, it is obvious that $T_{g}$ is bounded, in the proof of Theorem 1 , we have got that $g \in \mathcal{B}^{\alpha}$.

Conversely, let $\left\{f_{j}\right\}$ be an uniformly bounded sequence in $B(p, q)$ which converges to zero uniformly on any compact subset of $B$ when $j \rightarrow \infty$. From Lemma 1, we know that $\left\|f_{j}\right\|_{\mathcal{B}^{\frac{n+1+q}{p}}} \leq c\left\|f_{j}\right\|_{B(p, q)}$, thus $\left\{f_{j}\right\}$ is uniformly bounded in $\mathcal{B}^{\frac{n+1+q}{p}}$. Then by the hypothesis and Lemma 3 , we get that

$$
\lim _{j \rightarrow \infty} \sup _{z \in B}\left|f_{j}(z)\right|=0
$$

From which we have

$$
\left\|T_{g} f_{j}\right\|_{\mathcal{B}^{\alpha}} \leq c \sup _{z \in B}\left(1-|z|^{2}\right)^{\alpha}\left|f_{j}(z) R g(z)\right| \leq c\|g\|_{\mathcal{B}^{\alpha}} \sup _{z \in B}\left|f_{j}(z)\right| \rightarrow 0,
$$

when $j \rightarrow \infty$. So $T_{g}$ is compact from Lemma 5 .

Case 2. If $\frac{n+1+q}{p}=1$, it is sufficient to prove

$$
\lim _{|z| \rightarrow 1}\left(1-|z|^{2}\right)^{\alpha}|\operatorname{Rg}(z)| \log \frac{1}{1-|z|^{2}}=0 .
$$

Suppose that $\lim _{|z| \rightarrow 1}\left(1-|z|^{2}\right)^{\alpha}|R g(z)| \log \frac{1}{1-|z|^{2}} \neq 0$. Then there exists $\varepsilon_{0}>0,\left\{z^{j}\right\} \in B$, such that

$$
\left(1-\left|z^{j}\right|^{2}\right)^{\alpha}\left|R g\left(z^{j}\right)\right| \log \frac{1}{1-\left|z^{j}\right|^{2}} \geq \varepsilon_{0} .
$$

Let

$$
f_{j}(z)=\left(\log \frac{1}{1-\left|z^{j}\right|^{2}}\right)^{-\frac{2}{p}}\left(\log \frac{1}{1-<z, z^{j}>}\right)^{1+\frac{2}{p}} .
$$

We have shown that $f_{j} \in B(p, q)$ with $\left\|f_{j}\right\|_{B(p, q)} \leq c$, and it is obvious that $f_{j} \rightarrow 0$ uniformly on any compact subset of $B$ as $j \rightarrow \infty$. While

$$
\begin{aligned}
\| T_{g} & f_{j} \|_{\mathcal{B}^{\alpha}} \\
& \geq\left(1-\left|z^{j}\right|^{2}\right)^{\alpha}\left|f_{j}\left(z^{j}\right)\right|\left|R g\left(z^{j}\right)\right| \\
& =\left\{\left(1-\left|z^{j}\right|^{2}\right)^{\alpha}\left|R g\left(z^{j}\right)\right| \log \frac{1}{1-\left|z^{j}\right|^{2}}\right\}\left|f_{j}\left(z^{j}\right)\right|\left(\log \frac{1}{1-\left|z^{j}\right|^{2}}\right)^{-1} \\
& \geq \varepsilon_{0}\left|f_{j}\left(z^{j}\right)\right|\left(\log \frac{1}{1-\left|z^{j}\right|^{2}}\right)^{-1} \\
& =\varepsilon_{0},
\end{aligned}
$$


then $\left\|T_{g} f_{j}\right\|_{\mathcal{B}^{\alpha}}$ doesn't tend to 0 when $j \rightarrow \infty$. It's a contraction by Lemma 5. So

$$
\lim _{|z| \rightarrow 1}\left(1-|z|^{2}\right)^{\alpha}|R g(z)| \log \frac{1}{1-|z|^{2}}=0 .
$$

Meanwhile, note that $\lim _{|z| \rightarrow 1} \log \frac{1}{1-|z|^{2}}=\infty$, it is easy to see that $\lim _{|z| \rightarrow 1}\left(1-|z|^{2}\right)^{\alpha}|R g(z)|=0$.

Therefore, we have

$$
\lim _{|z| \rightarrow 1}\left(1-|z|^{2}\right)^{\alpha}|\operatorname{Rg}(z)| \log \frac{2}{1-|z|^{2}}=0 .
$$

If $\frac{n+1+q}{p}>1$, then consider

$$
f_{j}(z)=\frac{1-\left|z^{j}\right|^{2}}{\left(1-<z, z^{j}>\right)^{\frac{n+1+q}{p}}}
$$

and use the same method as in the case of $\frac{n+1+q}{p}=1$, we can also prove

$$
\lim _{|z| \rightarrow 1}\left(1-|z|^{2}\right)^{\alpha}|R g(z)| \log \frac{1}{1-|z|^{2}}=0 .
$$

Conversely, let $\left\{f_{j}\right\}$ be an uniformly bounded sequence in $B(p, q)$ which converges to zero uniformly on any compact subset of $B$ when $j \rightarrow \infty$. If $\lim _{|z| \rightarrow 1^{-}}\left(1-|z|^{2}\right)^{\alpha} G_{\frac{n+1+q}{p}}(z)|R g(z)|=0$, then for all $\varepsilon>0$, there exists $r \in(0,1)$, such that

$$
\left(1-|z|^{2}\right)^{\alpha} G_{\frac{n+1+q}{p}}(z)|R g(z)|<\varepsilon, r<|z|<1 .
$$

Then

$$
\begin{aligned}
& \left\|T_{g} f_{j}\right\|_{\mathcal{B}^{\alpha}} \\
& \leq \quad c \sup _{|z| \leq r}\left(1-|z|^{2}\right)^{\alpha}\left|f_{j}(z) R g(z)\right|+c \sup _{r<|z|<1}\left(1-|z|^{2}\right)^{\alpha}\left|f_{j}(z) R g(z)\right| \\
& \leq \quad c \sup _{|z| \leq r}\left(1-|z|^{2}\right)^{\alpha}|R g(z)|\left|f_{j}(z)\right| \\
& \quad+c \sup _{r<|z|<1}\left(1-|z|^{2}\right)^{\alpha} G_{\frac{n+1+q}{p}}(z)|R g(z)|\left\|f_{j}\right\|_{B(p, q)} \\
& \quad \quad \quad \quad \sup _{|z| \leq r}\left(1-|z|^{2}\right)^{q \alpha}\left|R g(z)\left\|f_{j}(z) \mid+c \varepsilon\right\| f_{j} \|_{B(p, q)}\right. \\
& \leq \quad c \varepsilon
\end{aligned}
$$

if $j$ is sufficiently large. It follows from Lemma 5 that $T_{g}$ is compact. So now, the proof of Theorem 2 is completed. 
Acknowledgements. The first author is partly supported by the National Natural Science Foundation of China (Grand Nos.10671141, 10371091). The authors would like to express their sincere thanks to the editor and referees for helpful comments and suggestions which improved the manuscript considerably.

\section{References}

[1] K. F. Andersen, Cesáro averaging operators on Hardy spaces, Proc. Royal Soc. Edinburgh, 126A (1996), 617-624.

[2] A. Aleman and A. Siskakis, Integration operators on Bergman spaces, Indiana Univ. Math. J., 46 (1997), 337-356.

[3] G. Benke and D. C. Chang, A note on weighted Bergman spaces and the cesáro operator, Nagoya Math. J., 159 (2000), 25-43.

[4] D. C. Chang and S. Stevic, The generalized Cesá ro operator on the unit polydisc, Taiwanese Jouirnal of Mathematics, 7 (1997), 293-308.

[5] N. Danikas and A. Siskakis, The Cesá ro operator on bounded analytic functions, Analysis, 13 (1993), 195-199.

[6] P. Durn, Theory of $H^{p}$ Space, Academic Press, New York, 1970.

[7] P. Galanopoulos, The Cesá ro operator on Dirichlet spaces, Acta Sci. Math., 67 (2001), 420-441.

[8] D.V. Giang and F. Morricz, The Cesáro operator on Dirichlet is bounded on the Hardy space $H^{1}$, Acta Sci. Math., 61 (2001), 535-544.

[9] Z.J. Hu, Extended Cesá ro operators on mixed norm space, Proc. Amer. Math. Soc., 131 (2001), 2171-2179.

[10] Z.J. Hu, Extended Cesáro operators on the Bloch spaces in the unit ball of $C^{n}$, Acta Math. Sci., 23B (2001), 561-566.

[11] Z.J. Hu, Extended Cesá ro operator on Bergman space, J. Math. Anal. Appl., 296 (2004), 435-454.

[12] S. Li and S. Stević, Riemann-Stieltjes operators on Hardy spaces in the unit ball of $C^{n}$, Bull. Belg. Math. Soc. Simon Stevin, 14 (2007), 621-628.

[13] S. Li and S. Stević, Riemann-Stieltjes type integral operators on the unit ball in $C^{n}$, Complex Variables Elliptic Functions, 52 (2007), 495-517.

[14] S. Li and S. Stević, Compactness of Riemann-Stieltjes operators between $F(p, q, s)$ and $\alpha$-Bloch spaces, Publ. Math. Debrecen, 72 (2008), 111-128. 
[15] S. Li and S. Stević, Riemann-Stieltjes operators between mixed norm spaces, Indian J. Math., 50 (2008), 177-188.

[16] S. Li and S. Stević, Volterra type operators on Zygmund space, J. Inequal. Appl., (2007), Article ID 32124, 10 pp.

[17] J. Miao, The cesáro operator is bounded on $H^{p}$ for $0<p<1$, Proc. Amer. Math. Soc., 116 (1992), 1077-1079.

[18] A. Siskakis, Composition semigroups and the Cesáro operator on $H^{p}(D)$, J. London Math. Soc., 36 (1987), 153-164.

[19] A. Siskakis, Semigroups of composition operators in Bergman spaces, Bull. Austral. Math. Soc., 35 (1987), 397-406.

[20] A. Siskakis, The cesá ro operator is bounded on $H^{1}$, Proc. Amer. Math. Soc., 110 (1990), 461-462.

[21] A. Siskakis, On the Bergman space norm of the cesá ro operator, Arch. Math., 67 (1996), 312-318.

[22] J. H. Shi and G. B. Ren, Boundedness of the cesá ro operator on mixed norm space, Proc. Amer. Math. Soc., 126 (1998), 3553-3560.

[23] K. Stempak, Cesá ro averaging operators, Proc. Royal. Soc. Edinburgh, 124A (1994), 121-126.

[24] J. Xiao, Cesáro-type operators on Hardy, BMOA and Bloch spaces, Arch. Math., 68 (1997), 398-406.

[25] J. Xiao, Riemann-Stieltjes operators on weighted Bloch and Bergman spaces of the unit ball, J. London Math. Soc., 70 (2004), 199-214.

[26] W. S. Yang and, C. H. Ouyang, Exact location of $\alpha-$ Bloch spaces in $L_{a}^{p}$ and $H^{p}$ of a complex unit ball, Rocky Mountain J. Math, 30 (2000), 1151-1169.

[27] Z.H. Zhou and R. Chen, Weighted composition operators fom $F(p, q, s)$ to Bloch type spaces, Int. J. Math., 19 (2008), 899-926.

[28] Z.H. Zhou, Composition Operators on the Lipschitz Spaces in Polydiscs, Science in China A, 46 (2003), 33-38.

[29] Z.H. Zhou and J.H. Shi, Compact composition operators on the Bloch spaces in polydiscs, Science in China (Series A), 44 (2001), 286-291.

[30] X.J. Zhang, Extended cesáro operators on Dirichlet type spaces and Bloch spaces of $C^{n}$, Chin. Ann. of Math.,(Series A), 26 (2005), 1391505.

[31] R. Zhao, On a general family of function spaces, Ann. Acad. Sci. Fenn. Math. Dissertationes, 105 (1996), 56 pp. 
[32] Z.H. Zhou and R. Chen, On the composition operators on the Bloch space of several complex variables, Science in China (Series A), 48 (2005), 392-399.

[33] Z.H. Zhou and Y. Liu, The essential norms of composition operators between generalized Bloch spaces in the polydisc and their applications, J. Ineq. Appl., (2006), Article ID 90742, 22pp.

[34] Z.H. Zhou and J.H. Shi, Compactness of composition operators on the Bloch space in classical bounded symmetric domains, Michigan Math. J., 50 (2002), 381-405.

[35] K.H. Zhu, Spaces of Holomorphic Functions in the Unit Ball, SpringerVerlag, 2004.

[36] K.H. Zhu, Operator Theory in Function Spaces, Pure and Applied Mathematics 136, Maecel Dekker, Inc., New York-Besel, 1990.

[37] W.X. Zhuo and C.H. Ouyang, Möbius invariant gradient and little $\alpha$-Bloch functions, Acta Mathematica Scientia, 22 (2002), 295-301.

Department of Mathematics,

Tianjin University

Tianjin 300072

P.R. China

(E-mail : zehuazhou2003@yahoo.com.cn)

(E-mail : loveminjie426@tom.com)

(Received : September 2006) 


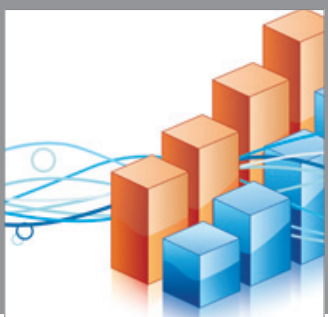

Advances in

Operations Research

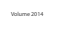

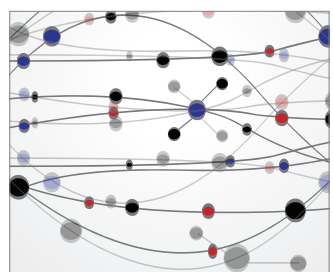

\section{The Scientific} World Journal
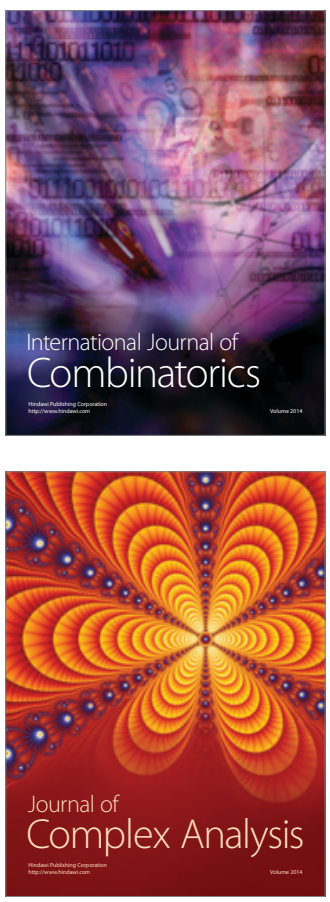

International Journal of

Mathematics and

Mathematical

Sciences
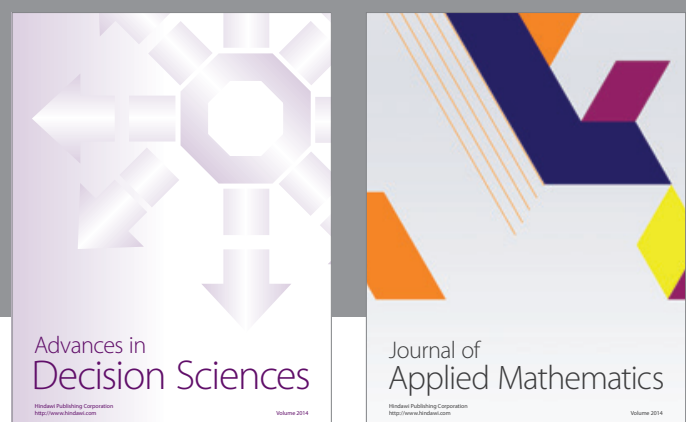

Journal of

Applied Mathematics
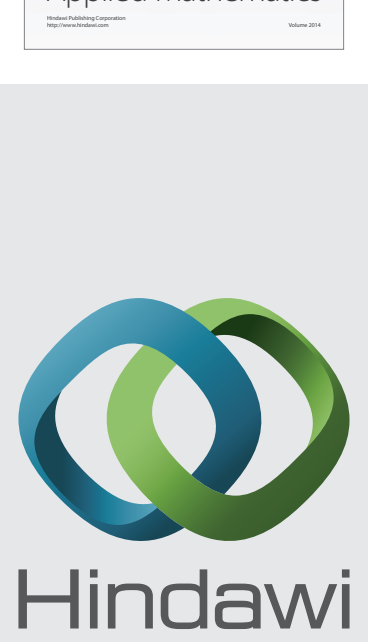

Submit your manuscripts at http://www.hindawi.com
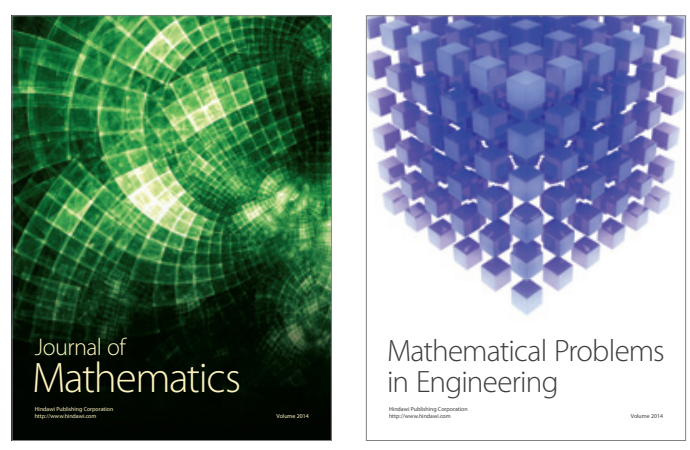

Mathematical Problems in Engineering
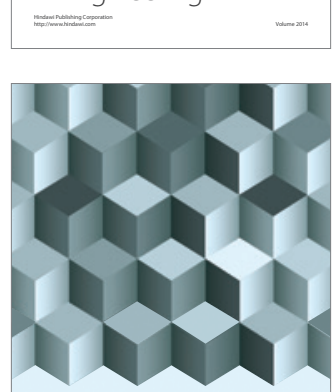

Journal of

Function Spaces
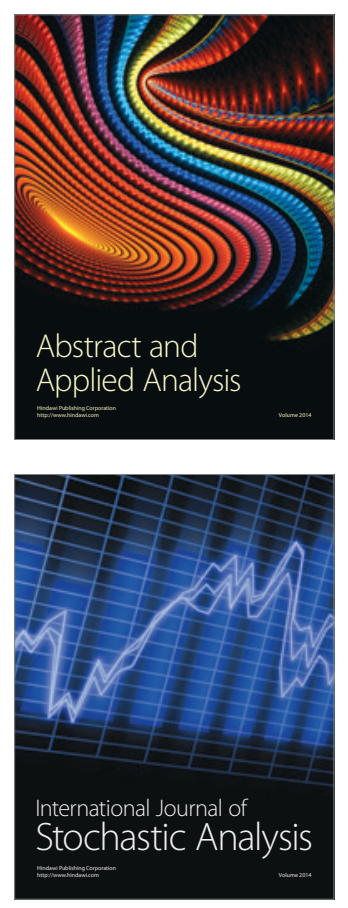

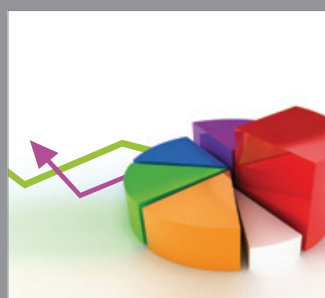

ournal of

Probability and Statistics

Promensencen
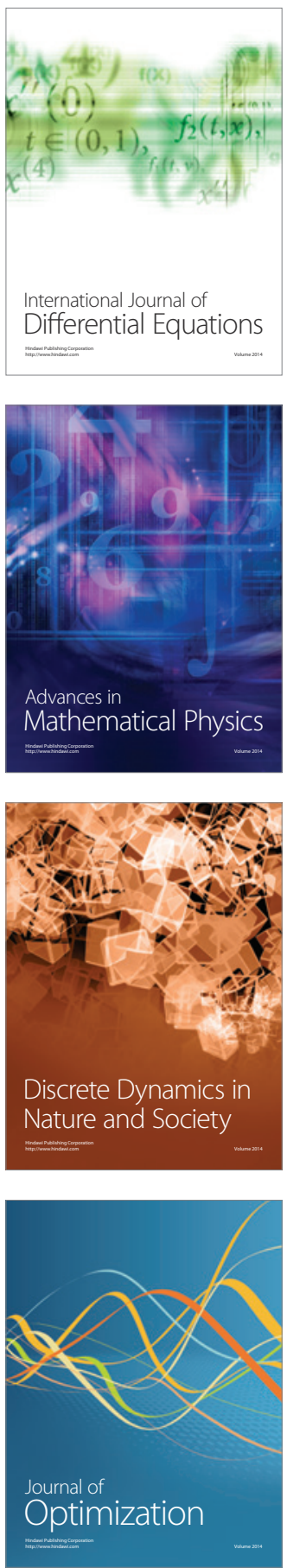